\title{
KL-6 in Obstructive Sleep Apnea: A Potential Biomarker of Subclinical Lung Injury
}

\author{
Sei Won Kim¹, Hwan Hee Kim¹, Heayon Lee', In Kyoung Kim¹, Sang Haak Lee1, and Hyeon Hui Kang² \\ 'Division of Pulmonary, Critical Care and Sleep Medicine, Department of Internal Medicine, Eunpyeong St. Mary's Hospital, \\ College of Medicine, The Catholic University of Korea, Seoul, Korea \\ ${ }^{2}$ Division of Pulmonary, Critical Care and Sleep Medicine, Department of Internal Medicine, Ulsan University Hospital, \\ University of Ulsan College of Medicine, Ulsan, Korea
}

\begin{abstract}
Objective: The relationship between obstructive sleep apnea (OSA) and cardiovascular diseases has been reported in several studies. However, little is known about the effects of OSA on pathophysiological processes in the lung. This study examined the association between OSA and serum levels of Krebs von den Lungen-6 (KL-6), which may reflect the degree of subclinical lung injury, according to the severity of OSA. Methods: Forty adults who were diagnosed with OSA using type 1 polysomnography were included in this study. Patients with pulmonary diseases, cardiovascular diseases except well-controlled hypertension, or other significant medical conditions were excluded. Results: Of the 40

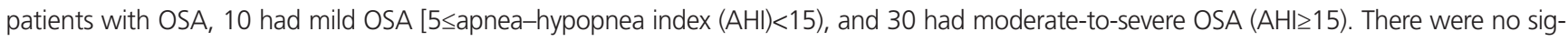
nificant differences in gender, age, smoking history, body mass index, neck circumference, and waist circumference between those with mild and those with moderate-to-severe OSA. The median serum level of KL-6 differed significantly between the mild and moderate-to-severe OSA groups $[180.8(135.3,213.8)$ vs. $281.5(171.3,410.6) \mathrm{U} / \mathrm{mL}, \mathrm{p}=0.018]$. After multiple regression analysis, the final model showed that smoking amount ( $b e t a=2.626, p=0.012$ ) and $A H I \geq 15$ (beta=2.256, $\mathrm{p}=0.031$ ) were significant predictors of KL-6. Conclusion: Serum KL-6 levels were significantly elevated in moderate-to-severe OSA compared with mild OSA, independent of smoking and other factors. OSA is associated with lung injury, and KL-6 shows potential as a clinical biomarker.
\end{abstract}

Key Words: Obstructive sleep apnea; Krebs von den Lungen-6; Lung injury; Hypoxia; Biomarker

Received: May 20, 2021 Accepted: June 8, 2021

Corresponding author: Hyeon Hui Kang, MD, PhD, Division of Pulmonary, Critical Care and Sleep Medicine, Department of Internal Medicine, Ulsan University Hospital, University of Ulsan College of Medicine, 877 Bangeojinsunhwando-ro, Dong-gu, Ulsan 44033, Korea.

Tel: 82-52-250-8660, Fax: 82-52-250-7048, E-mail: 0734950@uuh.ulsan.kr

(ac) This is an Open Access article distributed under the terms of the Creative Commons Attribution Non-Commercial License (https://creativecommons.org/licenses/bync/4.0) which permits unrestricted non-commercial use, distribution, and reproduction in any medium, provided the original work is properly cited.

\section{INTRODUCTION}

Obstructive sleep apnea (OSA) is characterized by repetitive upper airway narrowing or collapse during sleep, which causes intermittent hypoxia and sleep fragmentation [1,2]. OSA affects approximately $3.2-4.5 \%$ of the Korean population and is associated with significant morbidity and mortality [3]. However, up to $80 \%$ of moderate or severe OSA cases are undiagnosed despite adequate access to medical services $[4,5]$.

The relationship between OSA and cardiovascular diseases has been reported in several studies [6-8]. However, little is known about the effects of OSA on pathophysiological processes in the lung [9-11]. Repeated mechanical trauma related to upper airway occlusion, accelerated systemic inflammation, and intermittent nocturnal hypoxia lead to increased airway inflammation, oxidative stress, and apoptosis $[9,12,13]$. Oxidative stress in the alveoli may lead to epithelial and endothelial cell injury and increase alveolar wall permeability in the lungs $[14,15]$.

Krebs von den Lungen-6 (KL-6) is a high-molecular-weight mucin-like glycoprotein produced by type II pneumocytes and bronchial epithelial cells [16]. Serum levels of KL-6, which may reflect the degree of subclinical lung injury, have been shown to have a possible association with OSA $[17,18]$. However, only a few studies have reported this association, and the number of subjects in each study was relatively small.

In this study, we examined the association between OSA and lung injury using KL-6 and assessed the usefulness of KL-6 as a clinical biomarker. 


\section{METHODS}

\section{Subjects}

A total of 73 patients over 18 years of age who were diagnosed with OSA at Eunpyeong St. Mary's Hospital using type 1 polysomnography (PSG) were originally recruited. PSG was performed in the sleep laboratory of Eunpyeong St. Mary's Hospital. The diagnosis and measurement of the severity of OSA were performed according to the definition of the American Academy of Sleep Medicine [1]. The exclusion criteria were the presence of pulmonary diseases such as asthma, chronic obstructive pulmonary disease (COPD), or interstitial lung disease; cardiovascular diseases except well-controlled hypertension; and other significant medical conditions such as diabetes mellitus, thyroid disease, liver disease, chronic kidney disease, and malignant tumors. After these exclusions, 40 patients were enrolled in the present study. Approval was obtained from the Institutional Review Board of Eunpyeong St. Mary's Hospital (PC20SISI0151). Written informed consent was obtained from all the participating patients.

\section{Clinical measurements}

Demographic data including gender, age, body mass index (BMI), neck circumference, and waist circumference were collected. Smoking status was assessed based on the amount of smoking. Never-smokers were defined as those who had smoked fewer than 100 cigarettes in their lifetime [19]. Ex-smokers were defined as those who had quit smoking more than 6 months before the survey [20]. Pack-years were used to determine the amount of smoking. The presence of hypertension as well as the systolic blood pressure (SBP) and diastolic blood pressure (DBP) before PSG were checked. Total sleep time, sleep efficiency, the apneahypopnea index (AHI), nadir peripheral capillary oxygen saturation $\left(\mathrm{SpO}_{2}\right)$, and mean $\mathrm{SpO}_{2}$ were determined based on the results of PSG. The Epworth Sleepiness Scale (ESS) was administered before PSG.

\section{KL-6 measurement}

Serum levels of KL- 6 were measured using monoclonal antibody-based commercial enzyme-linked immunosorbent assay kits (MyBioSource, San Diego, CA, USA) according to the manufacturer's instructions. Results were obtained using an automated immunoassay system (Multimode microplate reader, Infinite M200 Pro, Tecan, Switzerland). KL-6 has been widely studied and used as a diagnostic and prognostic biomarker for interstitial lung disease. However, serum levels of KL-6 are also elevated in a variety of respiratory and non-respiratory conditions, including breast and pancreatic cancer and diabetes mellitus [21].

\section{Data analysis and statistics}

The mean and standard deviation were computed for normally distributed continuous variables, and the median and interquartile range (IQR; 25th to 75th percentile) for non-normally distributed continuous data. Categorical data are presented as numbers and percentages. To compare clinical data between subgroups, Student's t-test was performed for normally distributed data, and the Mann-Whitney U-test for non-normally distributed data. Categorical variables were compared using the chi-square or Fisher's exact test, as appropriate. Missing values were excluded from the analyses. Simple and multiple linear regression analyses were performed to identify independent clinical predictors of KL-6. Clinical parameters with p-values $<0.2$ on simple regression analysis were included in the multiple linear regression analysis. Stepwise backward elimination and all-subsets regression were performed to select the final model. Statistical analyses were performed using R software (ver. 3.5.3; R Core Team, R Foundation for Statistical Computing, Vienna, Austria). In all analyses, $p<0.05$ was taken to indicate statistical significance.

\section{RESULTS}

\section{Basic patient characteristics}

Table 1 summarizes the basic patient characteristics. Of the 40 patients with OSA, 10 had mild OSA $(5 \leq \mathrm{AHI}<15)$, and 30 had moderate-to-severe OSA (AHI $\geq 15)$. The percentage of males was $72.5 \%$. The mean age and median BMI were $53.6 \pm 17.1$ years and $27.7(24.9,30.4) \mathrm{kg} / \mathrm{m}^{2}$, respectively. There were no significant differences in gender, age, BMI, neck circumference, and waist circumference between the mild and moderate-to-severe OSA groups. Similarly, there were no significant differences in smoking status or the total amount of smoking between the mild and moderate-to-severe OSA groups. Among all subjects, $37.5 \%$ had hypertension. The mean SBP and DBP were $124.2 \pm 11.3 \mathrm{~mm} \mathrm{Hg}$ and $82.0 \pm 10.2 \mathrm{~mm} \mathrm{Hg}$, respectively. In addition, there were no significant differences in the percentage of patients with hypertension or the SBP or DBP between the mild and moderate-to-severe OSA groups. PSG data showed that the mean AHI scores in the mild and moderate-to-severe OSA groups were $8.7 \pm 4.6$ and $46.4 \pm 24.6$, respectively $(\mathrm{p}<0.001)$. The median nadir $\mathrm{SpO}_{2}$ during sleep was $88.5 \%(88.0,91.0)$ and $81.5 \%(76.0,86.0)$ in the mild and moderate-to-severe OSA groups, respectively $(\mathrm{p}<0.001)$. There was no significant difference in ESS scores between the two groups. However, the median serum level of KL-6 differed significantly between the mild and moderate-to-severe OSA groups [180.8 $(135.3,213.8) \mathrm{U} / \mathrm{mL}$ and $281.5(171.3,410.6) \mathrm{U} / \mathrm{mL}$, respectively; $\mathrm{p}=0.018]$ (Figure 1A).

\section{Basic patient characteristics according to gender}

Table 2 shows the basic characteristics of subjects according to gender. Although the serum level of KL-6 did not differ significantly between the mild and moderate-to-severe OSA groups for each gender, higher values were obtained for the moderate-to-severe OSA group compared to the mild OSA group. Other indicators exhibited the same trends as before the analysis according to gender. 
Clinical parameters associated with KL-6 in patients with OSA

Multiple linear regression analysis was performed to assess the associations of serum KL-6 with clinical parameters including AHI (Table 3). After simple regression analysis, gender, neck circumference, waist circumference, smoking amount, AHI (Figure
1B), and moderate-to-severe OSA were associated with p-values $<0.2$. After multiple regression analysis, the final model showed that smoking amount ( $b e t a=2.626, p=0.012$ ) and the presence of moderate-to-severe OSA (beta $=2.256, p=0.031$ ) were significant predictors of the KL-6 level.

Table 1. Basic characteristics of the study subjects

\begin{tabular}{|c|c|c|c|}
\hline Characteristics & Mild OSA $(n=10)$ & Moderate-to-severe OSA $(n=30)$ & p-value \\
\hline Male & $5(50.0)$ & $24(80.0)$ & 0.152 \\
\hline Age (years) & $55.8 \pm 21.1$ & $52.8 \pm 15.9$ & 0.642 \\
\hline $\mathrm{BMI}\left(\mathrm{kg} / \mathrm{m}^{2}\right)$ & $26.9[22.9,28.7]$ & $29.0[25.5,30.4]$ & 0.310 \\
\hline Neck circumference $(\mathrm{cm})$ & $38.5[34.0,41.0]$ & $38.0[37.0,41.0]$ & 0.686 \\
\hline Waist circumference $(\mathrm{cm})$ & $98.5[90.0,104.0]$ & $98.0[90.0,110.0]$ & 0.479 \\
\hline Smoking & & & 0.505 \\
\hline Never-smoker & $6(60.0)$ & $13(43.3)$ & \\
\hline Current smoker & $1(10.0)$ & $8(26.7)$ & \\
\hline Ex-smoker & $3(30.0)$ & $9(30.0)$ & \\
\hline Amount of smoking (PYRS) & $0.0[0.0,20.0]$ & $8.5[0.0,15.0]$ & 0.765 \\
\hline Hypertension & $3(30.0)$ & $12(40.0)$ & 0.850 \\
\hline $\mathrm{SBP}(\mathrm{mm} \mathrm{Hg})$ & $128.2 \pm 11.1$ & $122.9 \pm 11.2$ & 0.200 \\
\hline $\mathrm{DBP}(\mathrm{mm} \mathrm{Hg})$ & $85.0 \pm 11.0$ & $80.9 \pm 9.9$ & 0.281 \\
\hline Total sleep time (min) & $340.9 \pm 58.1$ & $313.7 \pm 62.9$ & 0.236 \\
\hline Sleep efficiency (\%) & $87.8[77.1,94.8]$ & $82.4[72.1,90.4]$ & 0.272 \\
\hline AHI (events/h) & $8.7 \pm 4.6$ & $46.4 \pm 24.6$ & $<0.001^{*}$ \\
\hline $\mathrm{SpO}_{2}$, nadir (\%) & $88.5[88.0,91.0]$ & $81.5[76.0,86.0]$ & $<0.001^{*}$ \\
\hline $\mathrm{SpO}_{2}$, mean $(\%)$ & $95.5[95.0,96.0]$ & $93.0[90.0,94.0]$ & $<0.001^{*}$ \\
\hline Epworth Sleepiness Scale & $9.3 \pm 4.9$ & $9.3 \pm 4.6$ & 0.985 \\
\hline Krebs von den Lungen-6 (U/mL) & $180.8[135.3,213.8]$ & $281.5[171.3,410.6]$ & $0.018^{*}$ \\
\hline
\end{tabular}

Data are presented as $\mathrm{n}(\%)$, mean \pm standard deviation, or median [interquartile range]. ${ }^{*} \mathrm{p}<0.05$. OSA: obstructive sleep apnea, BMI: body mass index, PYRS: pack-years, SBP: systolic blood pressure, DBP: diastolic blood pressure, AHI: apnea-hypopnea index
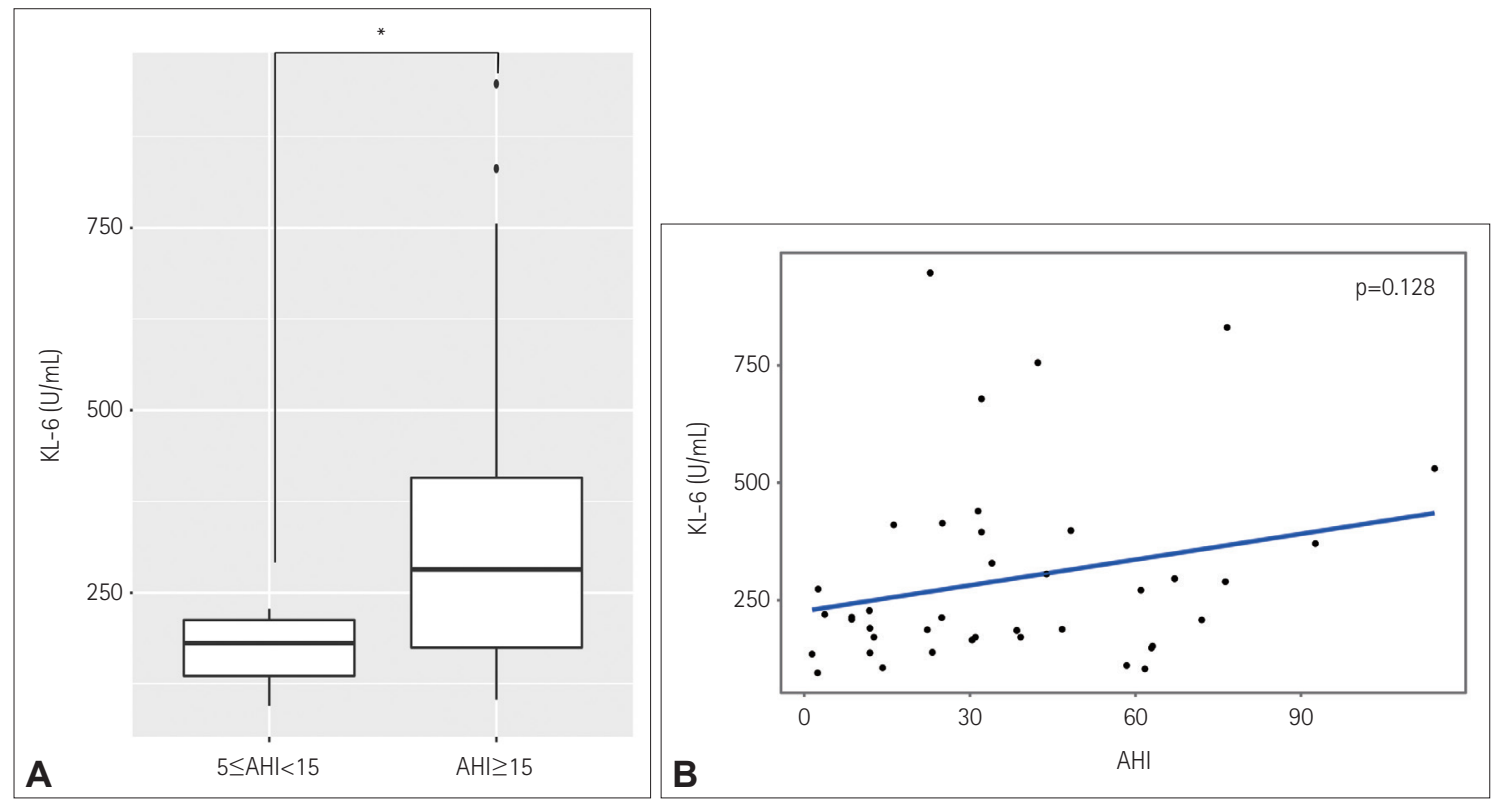

Figure 1. Relationship between serum KL-6 and AHI in patients with OSA. A: Box plots of serum KL-6 levels in the mild and moderateto-severe OSA groups. The median serum level of KL-6 differed significantly between the mild and moderate-to-severe OSA groups $[180.8(135.3,213.8)$ vs. $281.5(171.3,410.6) \mathrm{U} / \mathrm{mL}, p=0.018]\left({ }^{*} p<0.05\right)$. B: Scatter plot with a regression line representing the relationship between the serum KL-6 level and the AHI. Each data point represents one study subject. There was no significant correlation between KL-6 and the AHI ( $p=0.128)$. KL-6: Krebs von den Lungen-6, OSA: obstructive sleep apnea, AHI: apnea-hypopnea index. 
Table 2. Basic characteristics of subjects according to gender

\begin{tabular}{|c|c|c|c|c|c|c|}
\hline & \multicolumn{3}{|c|}{ Female } & \multicolumn{3}{|c|}{ Male } \\
\hline & $\begin{array}{l}\text { Mild OSA } \\
(\mathrm{n}=5)\end{array}$ & $\begin{array}{l}\text { Moderate-to-severe } \\
\text { OSA }(n=6)\end{array}$ & $\mathrm{p}$-value & $\begin{array}{l}\text { Mild OSA } \\
(\mathrm{n}=5)\end{array}$ & $\begin{array}{l}\text { Moderate-to-severe } \\
\text { OSA }(n=24)\end{array}$ & p-value \\
\hline Age (years) & $56.2 \pm 23.7$ & $65.8 \pm 10.5$ & 0.391 & $55.4 \pm 21.1$ & $49.6 \pm 15.5$ & 0.479 \\
\hline $\mathrm{BMI}\left(\mathrm{kg} / \mathrm{m}^{2}\right)$ & $26.7 \pm 7.2$ & $27.4 \pm 2.3$ & 0.852 & $27.5[27.2,28.7]$ & $29.5[25.6,31.8]$ & 0.603 \\
\hline Smoking & & & 1.000 & & & 0.535 \\
\hline Never smoker & $5(100.0)$ & $5(83.3)$ & & $1(20.0)$ & $8(33.3)$ & \\
\hline Current smoker & $0(0.0)$ & $0(0.0)$ & & $1(20.0)$ & $8(33.3)$ & \\
\hline Ex-smoker & $0(0.0)$ & $1(16.7)$ & & $3(60.0)$ & $8(33.3)$ & \\
\hline Amount of smoking (PYRS) & $0.0[0.0,0.0]$ & $0.0[0.0,0.0]$ & 0.465 & $20.0[15.0,20.0]$ & $10.0[0.0,20.0]$ & 0.213 \\
\hline Neck circumference $(\mathrm{cm})$ & $35.0 \pm 2.5$ & $35.7 \pm 1.9$ & 0.628 & $41.0[40.0,43.0]$ & $39.0[38.0,41.5]$ & 0.237 \\
\hline Waist circumference $(\mathrm{cm})$ & $93.8 \pm 10.9$ & $94.3 \pm 6.8$ & 0.923 & $104.0[97.0,104.0]$ & $102.0[90.5,111.0]$ & 0.719 \\
\hline Hypertension & $2(40.0)$ & $5(83.3)$ & 0.391 & $1(20.0)$ & $7(29.2)$ & $>0.999$ \\
\hline $\mathrm{SBP}(\mathrm{mm} \mathrm{Hg})$ & $124.0[120.0,130.0]$ & $120.0[120.0,130.0]$ & 0.922 & $130.0[128.0,140.0]$ & $120.0[119.0,130.0]$ & 0.088 \\
\hline DBP (mm Hg) & $79.2 \pm 10.2$ & $81.7 \pm 7.5$ & 0.654 & $90.8 \pm 9.1$ & $80.7 \pm 10.5$ & 0.059 \\
\hline Total sleep time (min) & $326.9 \pm 72.3$ & $305.8 \pm 74.3$ & 0.647 & $354.9 \pm 43.3$ & $315.7 \pm 61.4$ & 0.188 \\
\hline Sleep efficiency (\%) & $82.3 \pm 13.9$ & $72.4 \pm 15.8$ & 0.307 & $90.6[85.1,94.8]$ & $84.0[78.2,91.0]$ & 0.295 \\
\hline AHI (events/h) & $8.1 \pm 5.5$ & $29.0 \pm 9.1$ & $0.002^{*}$ & $9.3 \pm 4.1$ & $51.1 \pm 24.8$ & $<0.001^{*}$ \\
\hline $\mathrm{SpO}_{2}$, nadir (\%) & $90.2 \pm 2.2$ & $83.8 \pm 3.7$ & $0.008^{*}$ & $88.0[88.0,89.0]$ & $79.0[75.5,86.0]$ & $0.026^{*}$ \\
\hline $\mathrm{SpO}_{2}$, mean $(\%)$ & $96.2 \pm 0.8$ & $93.8 \pm 2.1$ & $0.046^{*}$ & $95.0[95.0,95.0]$ & $93.0[90.0,93.5]$ & $0.010^{*}$ \\
\hline Epworth Sleepiness Scale & $8.0[7.0,10.0]$ & $6.0[6.0,7.0]$ & 0.160 & $8.2 \pm 3.6$ & $9.8 \pm 4.7$ & 0.484 \\
\hline Krebs von den Lungen- $6(\mathrm{U} / \mathrm{mL})$ & $168.3 \pm 48.2$ & $252.4 \pm 119.1$ & 0.175 & $190.3[137.7,213.8]$ & $292.6[179.1,426.6]$ & 0.068 \\
\hline
\end{tabular}

Data are presented as $\mathrm{n}(\%)$, mean $\pm \mathrm{SD}$, or median [interquartile range]. ${ }^{*} \mathrm{p}<0.05$. OSA: obstructive sleep apnea, BMI: body mass index, PYRS: packyears, SBP: systolic blood pressure, DBP: diastolic blood pressure, AHI: apnea-hypopnea index.

Table 3. Clinical parameters associated with Krebs von den Lungen- 6 in patients with OSA

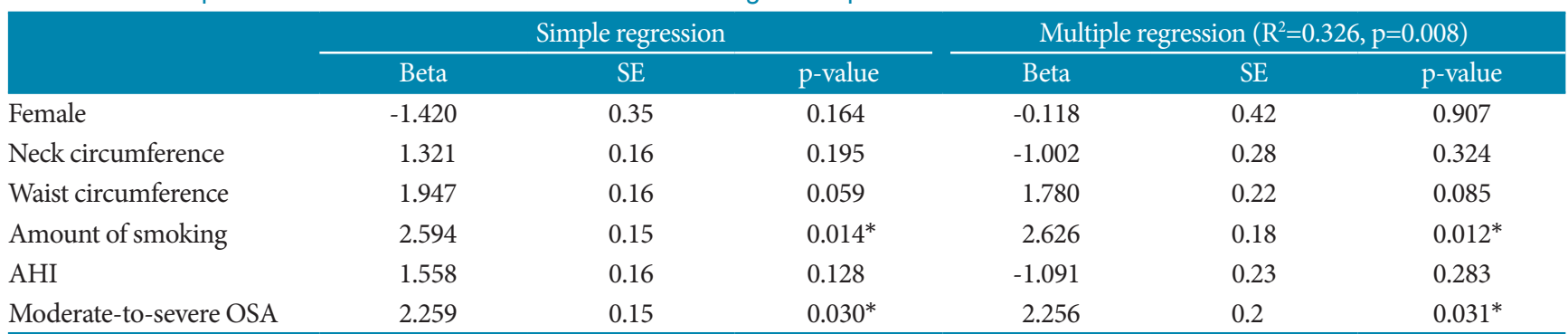

${ }^{*} \mathrm{p}<0.05$. $\mathrm{R}^{2}$ : adjusted $\mathrm{R}^{2}$ from the multiple linear regression model, SE: standard error, AHI: apnea-hypopnea index, OSA: obstructive sleep apnea

\section{DISCUSSION}

In this study, we examined the potential of KL- 6 as a biomarker of subclinical lung injury in patients with OSA. Our results revealed significantly higher serum KL-6 levels in patients with moderate-to-severe OSA than in those with mild OSA. Similar trends were observed in subgroup analysis based on gender. After adjusting for other factors, the amount of smoking and the presence of moderate-to-severe OSA were significantly related to serum KL-6 levels in OSA patients.

OSA is well known for its numerous health outcomes and physiological effects, particularly cardiovascular disease [6,22]. Intermittent hypoxemia (IH), sympathetic activation, endothelial dysfunction, a depressed baroreflex sensitivity, and increased platelet aggregability are proposed mechanisms related to increased cardiovascular morbidity in OSA patients [22]. However, little is known about the effects of OSA on pathophysiological changes in the lung. Intrathoracic pressure swings, $\mathrm{IH}$, and repeated collapse of the upper airway affect the structure and function of the respiratory tract, resulting in local inflammation, remodeling, and hyperreactivity [23-25]. IH likely leads to oxidative stress and generates reactive oxygen species, which may play an important role in activating the inflammatory response [26]. Animal studies have shown that IH exposure results in lung damage caused by oxidative stress, inflammation, and apoptosis $[9,13]$. In patients with severe OSA, the level of nitric oxide in exhaled breath, which is an important mediator of airway inflammation, was higher compared to that in the controls [27].

The effects of OSA on pulmonary diseases such as COPD and idiopathic pulmonary fibrosis (IPF) have begun to be studied. The presence of both OSA and COPD (the so-called "overlap syndrome") causes more severe nocturnal hypoxemia and a greater risk for morbidity and mortality compared to either COPD or OSA alone $[28,29]$. The worsening impact of OSA in patients 
with IPF has also been suggested in several clinical and experimental studies [30,31]. Taken together, these findings indicate that OSA has a significant impact on lung health.

KL-6 has been reported as a sensitive marker for interstitial lung diseases such as IPF, connective tissue disease-associated interstitial lung disease, hypersensitivity pneumonitis, and pulmonary sarcoidosis [32]. Increased levels of KL-6 reflect the extent of lung damage and regeneration of type II pneumocytes. A few studies have investigated the association between serum KL- 6 and OSA. Lederer et al. [18] checked the plasma KL-6 levels in 11 OSA and 10 control patients. Although the sample size was limited, they found median KL-6 levels of $317(232,506) \mathrm{U} / \mathrm{mL}$ in the OSA group and $226(179,257) \mathrm{U} / \mathrm{mL}$ in the control group $(\mathrm{p}=0.003)$. Aihara et al. [17] showed that the AHI was significantly positively correlated with serum KL-6 levels even after adjustment for BMI and smoking $(\mathrm{p}=0.03)$. However, with nonsmoking subjects, Liang et al. [33] reported no significant difference in KL-6 between groups with high hypopnea index scores and those with low scores. In our study, the serum level of KL-6 was significantly higher in patients with moderate-to-severe OSA compared to those with mild OSA. After adjusting for other significant factors including smoking, moderate-to-severe OSA was a significant independent predictor of KL-6 levels. However, there was no significant relationship between the AHI and KL-6 in our study. Patients with OSA are often heterogenous, and this heterogeneity is not captured by $\mathrm{AHI}$ alone. In addition, the nadir $\mathrm{SpO}_{2}$ and mean $\mathrm{SpO}_{2}$ were not significantly correlated with KL-6.

Our study has several limitations. First, the study population was small. Serum levels of KL-6 are influenced by a variety of respiratory and non-respiratory conditions. To exclude the effects of factors other than OSA, we excluded OSA patients who had pulmonary diseases, cardiovascular diseases except well-controlled hypertension, or significant comorbidities such as diabetes. Thus, many OSA patients who had comorbid conditions were excluded from the study. Second, we did not directly check the severity of lung injury, such by measuring cell counts, cytokines, and KL-6 in bronchoalveolar lavage fluid. Although serum KL-6 reflects the extent of lung damage, it is not always possible to conclude that there is a clear lung injury based on the KL-6 level. Third, presence of moderate-to-severe OSA was a significant independent predictor of serum KL-6 in this study. However, there was no significant relationship between the AHI and KL-6. The relationships between various sleep disorder indicators and KL-6 should be studied further.

In summary, serum KL-6 was significantly elevated in patients with moderate-to-severe OSA compared to those with mild OSA, independent of smoking and other factors. This study showed that OSA affects lung injury and that KL-6 may be a useful clinical biomarker in OSA patients. Further studies are needed to identify lung-specific biomarkers and detailed mechanisms in OSA.

\section{Acknowledgments}

None

\section{Conflicts of Interest}

The authors have no potential conflicts of interest to disclose.

\section{Author Contributions}

Conceptualization: Sei Won Kim, Sang Haak Lee, Hyeon Hui Kang. Formal analysis: Hwan Hee Kim, Heayon Lee, In Kyoung Kim, Sei Won Kim. Methodology: In Kyoung Kim, Sei Won Kim. Project administration: Hyeon Hui Kang. Resources: Hwan Hee Kim, Heayon Lee, Sei Won Kim, Sang Haak Lee, Hyeon Hui Kang. Writing_-original draft: Sei Won Kim. Writing_review \& editing: Sei Won Kim, Sang Haak Lee, Hyeon Hui Kang.

\section{ORCID iDs}

Sei Won Kim (D)

https://orcid.org/0000-0002-2798-421X

Hwan Hee Kim (D)

https://orcid.org/0000-0003-1984-1371

Heayon Lee (D)

https://orcid.org/0000-0001-7389-2343

In Kyoung Kim (D)

https://orcid.org/0000-0001-9975-8417

Sang Haak Lee (ib)

https://orcid.org/0000-0001-6259-7656

Hyeon Hui Kang (10)

https://orcid.org/0000-0001-5860-9407

\section{REFERENCES}

1. Sleep-related breathing disorders in adults: recommendations for syndrome definition and measurement techniques in clinical research. The Report of an American Academy of Sleep Medicine Task Force. Sleep 1999;22:667-689.

2. Eckert DJ, Malhotra A. Pathophysiology of adult obstructive sleep apnea. Proc Am Thorac Soc 2008;5:144-153.

3. Kim J, In K, Kim J, You S, Kang K, Shim J, et al. Prevalence of sleep-disordered breathing in middle-aged Korean men and women. Am J Respir Crit Care Med 2004;170:1108-1113

4. Kapur V, Strohl KP, Redline S, Iber C, O'Connor G, Nieto J. Underdiagnosis of sleep apnea syndrome in U.S. communities. Sleep Breath 2002;6:49-54.

5. Young T, Evans L, Finn L, Palta M. Estimation of the clinically diagnosed proportion of sleep apnea syndrome in middle-aged men and women. Sleep 1997;20:705-706.

6. Drager LF, McEvoy RD, Barbe F, Lorenzi-Filho G, Redline S; INCOSACT Initiative (International Collaboration of Sleep Apnea Cardiovascular Trialists). Sleep apnea and cardiovascular disease: lessons from recent trials and need for team science. Circulation 2017;136:1840-1850.

7. Molnar MZ, Mucsi I, Novak M, Szabo Z, Freire AX, Huch KM, et al. Association of incident obstructive sleep apnoea with outcomes in a large cohort of US veterans. Thorax 2015;70:888-895.

8. Hung J, Whitford EG, Parsons RW, Hillman DR. Association of sleep apnoea with myocardial infarction in men. Lancet 1990;336:261-264.

9. Tuleta I, Stöckigt F, Juergens UR, Pizarro C, Schrickel JW, Kristiansen G, et al. Intermittent hypoxia contributes to the lung damage by increased oxidative stress, inflammation, and disbalance in protease/antiprotease system. Lung 2016;194:1015-1020.

10. Lee EJ, Heo W, Kim JY, Kim H, Kang MJ, Kim BR, et al. Alteration of inflammatory mediators in the upper and lower airways under chronic intermittent hypoxia: preliminary animal study. Mediators Inflamm 2017;2017: 4327237.

11. Zhang SD, Wang P, Zhang J, Wang W, Yao LP, Gu CB, et al. 2'-O-galloylhyperin attenuates LPS-induced acute lung injury via up-regulation antioxidation and inhibition of inflammatory responses in vivo. Chem Biol Inter- 
act 2019;304:20-27.

12. Sabato R, Guido P, Salerno FG, Resta O, Spanevello A, Barbaro MP. Airway inflammation in patients affected by obstructive sleep apnea. Monaldi Arch Chest Dis 2006;65:102-105.

13. da Rosa DP, Forgiarini LF, Baronio D, Feijó CA, Martinez D, Marroni NP. Simulating sleep apnea by exposure to intermittent hypoxia induces inflammation in the lung and liver. Mediators Inflamm 2012;2012:879419.

14. Cochrane CG, Spragg R, Revak SD. Pathogenesis of the adult respiratory distress syndrome. Evidence of oxidant activity in bronchoalveolar lavage fluid. J Clin Invest 1983;71:754-761.

15. Kiefmann R, Rifkind JM, Nagababu E, Bhattacharya J. Red blood cells induce hypoxic lung inflammation. Blood 2008;111:5205-5214.

16. Frix AN, Schoneveld L, Ladang A, Henket M, Duysinx B, Vaillant F, et al. Could KL-6 levels in COVID-19 help to predict lung disease? Respir Res 2020;21:309.

17. Aihara K, Oga T, Harada Y, Chihara Y, Handa T, Tanizawa K, et al. Comparison of biomarkers of subclinical lung injury in obstructive sleep apnea. Respir Med 2011;105:939-945.

18. Lederer DJ, Jelic S, Basner RC, Ishizaka A, Bhattacharya J. Circulating KL6, a biomarker of lung injury, in obstructive sleep apnoea. Eur Respir J 2009; 33:793-796

19. Bondy SJ, Victor JC, Diemert LM. Origin and use of the 100 cigarette criterion in tobacco surveys. Tob Control 2009;18:317-323.

20. Lee YI, Lee SG, Kang DM, Kim JE, Kim YK, Leem JH, et al. Work-relatedness of lung cancer by smoking and histologic type in Korea. Ann Occup Environ Med 2014;26:43.

21. Sato H, Callister ME, Mumby S, Quinlan GJ, Welsh KI, duBois RM, et al. KL-6 levels are elevated in plasma from patients with acute respiratory distress syndrome. Eur Respir J 2004;23:142-145.

22. Peker Y, Hedner J, Norum J, Kraiczi H, Carlson J. Increased incidence of cardiovascular disease in middle-aged men with obstructive sleep apnea: a 7-year follow-up. Am J Respir Crit Care Med 2002;166:159-165.

23. Sarıman N, Levent E, Cubuk R, Yurtlu S, Benli Aksungar F. Bronchial hyperreactivity and airway wall thickening in obstructive sleep apnea patients. Sleep Breath 2011;15:341-350.

24. Kimoff RJ, Hamid Q, Divangahi M, Hussain S, Bao W, Naor N, et al. Increased upper airway cytokines and oxidative stress in severe obstructive sleep apnoea. Eur Respir J 2011;38:89-97.

25. Wasicko MJ, Erlichman JS, Leiter JC. Control of segmental upper airway resistance in patients with obstructive sleep apnea. J Appl Physiol (1985) 1993;74:2694-2703.

26. Lavie L. Sleep-disordered breathing and cerebrovascular disease: a mechanistic approach. Neurol Clin 2005;23:1059-1075.

27. Fortuna AM, Miralda R, Calaf N, González M, Casan P, Mayos M. Airway and alveolar nitric oxide measurements in obstructive sleep apnea syndrome. Respir Med 2011;105:630-636.

28. Owens RL, Malhotra A. Sleep-disordered breathing and COPD: the overlap syndrome. Respir Care 2010;55:1333-1346.

29. Donovan LM, Feemster LC, Udris EM, Griffith MF, Spece LJ, Palen BN, et al. Poor outcomes among patients with chronic obstructive pulmonary disease with higher risk for undiagnosed obstructive sleep apnea in the LOTT cohort. J Clin Sleep Med 2019;15:71-77.

30. Gille T, Didier M, Rotenberg C, Delbrel E, Marchant D, Sutton A, et al. Intermittent hypoxia increases the severity of bleomycin-induced lung injury in mice. Oxid Med Cell Longev 2018;2018:1240192.

31. Mermigkis C, Bouloukaki I, Schiza SE. Sleep as a new target for improving outcomes in idiopathic pulmonary fibrosis. Chest 2017;152:1327-1338.

32. Tzouvelekis A, Kouliatsis G, Anevlavis S, Bouros D. Serum biomarkers in interstitial lung diseases. Respir Res 2005;6:78.

33. Liang S, Li N, Heizhati M, Yao X, Abdireim A, Wang Y, et al. What do changes in concentrations of serum surfactant proteins A and D in OSA mean? Sleep Breath 2015;19:955-962. 